V. Zusammenfassung und Schlußbemerkung

Die Diskussion der verschiedenen Einflüsse, welche die Empfindlichkeit eines $1 / v$-Neutronenzählers mit Bremsmantel begrenzen, führte auf ein Rechenschema zur Bestimmung der spektralen Empfindlichkeit. Die numerische Auswertung ist durch folgende Stufen gekennzeichnet:

1. Bestimmung der Wahrscheinlichkeit $W\left(E_{0}\right)$, daß Neutronen des Energiebereiches zwischen $E_{0}$ und $E_{0}+\mathrm{d} E_{0}$ innerhalb des Bremsmantels thermisch werden nach $\mathrm{Gl} .(7-0)$.

2. Korrektur von $W\left(E_{0}\right)$ auf $W_{c}\left(E_{0}\right)$ für eine endliche Länge des Zählers nach Gl. (10-0).

3. Normierung der zylindersymmetrischen Quellenverteilung [Gl. (11-4)] mit $W_{c}\left(E_{0}\right)$ durch Gl. $(11-6)$.

4. Ausrechnung von $\psi\left(r_{q}\right)$.

5. Graphische Integration der mit $\psi\left(r_{q}\right)$ reduzierten Quellstärke über den Bereich des Bremsmantels.
Wir möchten annehmen, daß die hier entwickelten Beziehungen über eine nur qualitative Beschreibung der spektralen Empfindlichkeit hinausgehen. Wieweit allerdings die quantitativen Angaben den tatsächlichen Verhältnissen nahe kommen, läßt sich an Hand empirischer Daten, die in der Literatur zugänglich sind, schwer abschätzen, da hierzu genauere Angaben über das benützte Neutronenspektrum und die Einstrahlungsgeometrie erforderlich sind. Den Nutzen unserer Überlegungen sehen wir vorläufig in den Hinweisen, die für die Dimensionierung von Zählern für gegebene Versuchsbedingungen aus den einzelnen Berechnungsstufen abzuleiten sind. Beispielsweise lassen sich die Verhältnisse des "langen Zählers“ (flat counter) nach einem ähnlichen Schema übersehen, wenn man die Zentren der „Bremsschalen“ auf die Stirnfläche der Paraffinhülle verlegt und das Diffusionsproblem für eine Abhängigkeit der Quellenverteilung vom Achsenabstand $r$ und vom Abstand $z$ von der Stirnfläche löst.

Eine eingehende experimentelle Prüfung der quantitativen Angaben ist in Vorbereitung, über deren Ergebnis zur gegebenen Zeit berichtet werden soll.

\title{
Berechnung des elektrostatischen Feldes eines Mehrdraht-Antikoinzidenz-Zählrohres
}

\begin{abstract}
Von Alfred Seeger
Aus dem Institut für theoretische und angewandte Physik der Technischen Hochschule Stuttgart

(Z. Naturforschg. 6 a, 688-694 [1951]; eingegangen am 9. August 1951)

Herrn Professor Erich Regener zum 70. Geburtstag

Das elektrostatische Feld eines Mehrdrahtantikoinzidenzzählrohres wird mit Hilfe von $\hat{v}$-Funktionen allgemein berechnet. Für ein spezielles Beispiel wird die Feldstärke in den Symmetrieebenen zwischen den Zähldrähten sowie der praktisch wichtige Feldstärkengradient in den dort auftretenden feldfreien Linien zahlenmäßig angegeben.
\end{abstract}

$A^{\text {ut }}$ uf Anregung von Schopper wurde, im Anschluß an Untersuchungen vonW e i $\beta^{1}$ über Mehrdrahtzählrohre mit ebenen Platten, von $\mathrm{J}$ ü r g e $\mathrm{n}$ s e $\mathrm{n}^{2}$ ein Antikoinzidenzzähler entworfen und gebaut, dessen Grundbestandteile zwei koaxial ineinandergestellte Zählrohre sind. Einzelheiten werden von den genannten Autoren publiziert ${ }^{3}$. In der vorliegenden Arbeit befassen wir uns lediglich mit der Berechnung des

1 H. M. W e i ß, Diplomarbeit T. H. Stuttgart 1950.

2 H. J ür g e n s e n, Diplomarbeit T. H. Stuttgart 1951.

3 H. J ür gensen u. H. M. Weiß, Z. Naturforschg. 6a, 694 [1951]. elektrostatischen Feldes im äußeren Zählrohr. Dessen Kenntnis ist für den zuverlässigen Betrieb des Rohres wichtig, da das Feld überall im Rohr genügend stark sein muß, um die Rekombination der durch einfallende geladene Teilchen gebildeten Ionen zu verhindern und im gesamten Zählvolumen konstante Ansprechwahrscheinlichkeit zu gewährleisten.

$\S 1$. Das idealisierte elektrostatische Problem

Abb. 1 gibt einen Schnitt durch das Zählrohr wieder. Innen- und Außenzylinder mit den Radien $a$ und 
$b$ befinden sich auf gleichem elektrostatischen Potential $^{4}$. Auf einem Kreis vom Radius $c$ sind $m$ Zähldrähte vom Durchmesser $d$ in der Art eines regulären $m$-Ecks angeordnet, die ein gemeinsames positives Potential $\Phi_{0}$ gegenüber den Zylindern besitzen. Bei dem von Jürgensen gebauten Zählrohr ist

der Außendurchmesser des Innenzylinders

$$
2 a=30 \mathrm{~mm},
$$

der Innendurchmesser des Außenzylinders

$$
\begin{aligned}
2 b & =87 \mathrm{~mm}, \\
d & =0,1 \mathrm{~mm} \\
m & =6 .
\end{aligned}
$$

der Zähldrahtdurchmesser

und die Zahl der Zähldrähte

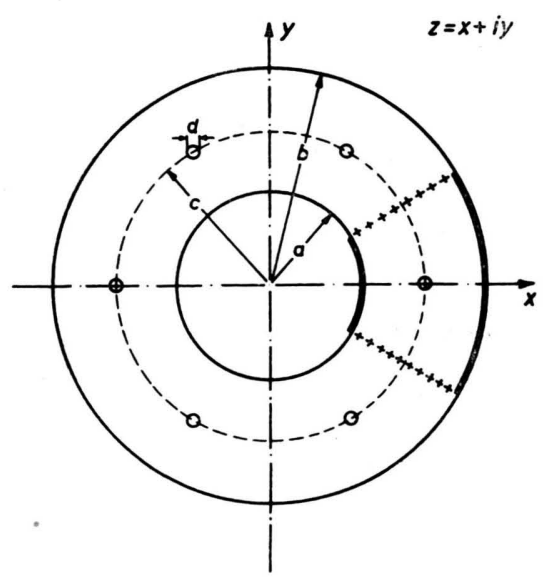

Abb. 1. Querschnitt durch das Zählrohr mit eingezeichneter komplexer Zahlenebene $\boldsymbol{z}$.

Für den Radius $c$ wurde aus technischen Gründen $c=1 / 2(a+b)$ gewählt. Wenn genügend Erfahrungen mit dem Betrieb des Zählrohres vorliegen, so lassen sich an Hand des bekannten elektrischen Feldes sicher auch physikalische Gesichtspunkte für eine zweckmäßige Wahl von $c$ angeben. Für die folgende Rechnung lassen wir deshalb den Wert von $c$ vorläufig noch offen.

Es soll nunmehr das elektrostatische Feld im Zwischengebiet der beiden Zylinder berechnet werden, solange die durch den Ionisierungsvorgang hervorgerufenen Raumladungen vernachlässigbar sind. Um funktionentheoretische Hilfsmittel anwenden zu können, wird das Problem als zweidimensional behandelt. Wir sehen also von der Tatsache ab, daß das Zählrohr eine endliche Länge in der Richtung senkrecht zur $x$ - $y$-Ebene hat. Dies ist durchaus gerechtfertigt, da beim Experiment nur der Mittelteil des Rohres be-

4 Für die Berechnung würde es keine Schwierigkeit machen, eine Potentialdifferenz zwischen diesen beiden Zylindern anzunehmen. nutzt wird, in dem der Randeinfluß wohl sicher vernachlässigbar ist.

Eine zweite, im vorliegenden Fall gleichfalls erlaubte Idealisierung ist die Vernachlässigung des Zähldrahtdurchmessers gegenüber den übrigen bei der Aufgabe auftretenden Entfernungen. Das bedeutet, daß wir darauf verzichten, die Drahtoberflächen exakt zu Äquipotentialflächen zu machen. Es werden vielmehr in den Drahtmittelpunkten Linienladungen $Q$ pro Längeneinheit des Drahtes angebracht, die von der Spannung $\Phi_{0}$ abhängen und die allein das Feld an der jeweiligen Drahtoberfläche bestimmen.

Das zu lösende idealisierte Problem lautet also: $\mathrm{Ge}-$ sucht ist das nur von den Koordinaten $x$ und $y$ abhängende Potential $\Phi$, das auf den beiden Zylinderoberflächen Null ist und das in den Zähldrahtmittellinien Linienladungen der Stärke $Q$ als Singularitäten besitzt.

Diese Aufgabe werden wir in $\S 2$ in geschlossener Form vollständig lösen. Man kann jedoch von der zuletzt genannten Idealisierung nötigenfalls auch absehen und wenigstens näherungsweise das Potential $\Phi$ so angeben, daß es auf den Kreiszylindern mit Radius $1 / 2 d$ um die Zähldrahtmittellinien den vorgeschriebenen Wert $\Phi_{0}$ annimmt. Da jedoch wegen der Kleinheit von $d$ diese Erweiterung ohne wesentliche Bedeutung ist, gehen wir darauf in $\$ 4$ nur kurz ein.

\section{$\S 2$ Feldbestimmung mit Hilfe der konformen Abbildung}

Das elektrostatische Potential $\Phi$, aus dem man die elektrische Feldstärke $\tilde{\mathfrak{E}}=-\operatorname{grad} \Phi$ erhält, genügt der Potentialgleichung

$$
\frac{\partial^{2} \Phi}{\partial x^{2}}+\frac{\partial^{2} \Phi}{\partial y^{2}}=0
$$

und kann deshalb als Realteil $\Phi(x, y)$ einer analytischen Funktion $Z=\Phi+i \Psi$ der komplexen Variablen $z=x+i y$ dargestellt werden. Aus dem komplexen Potential $Z(z)$ erhält man die elektrische Feldstärke, als Vektor in der komplexen Ebene aufgefaßt, nach der Formel

$$
\leftleftarrows=E_{x}+i E_{y}=-\left(\frac{\mathrm{d} Z}{\mathrm{~d} z}\right)^{*}
$$

(* = konjugiert komplex).

Zur Lösung der in $\S 1$ formulierten Randwertaufgabe für $\Phi$ verwenden wir die konforme Abbildung. Durch die Transformation

und

$$
z_{1}=(z / c)^{m}
$$

$$
z_{2}=-i \ln z_{1}=-i m \ln (z / c)
$$


entstehen aus dem in Abb. 1 eingezeichneten Kreisringsektor vom Öffnungswinkel $2 \pi / m$ die in Abb. 2 und 3 gezeichneten Gebiete. Die Lösung der Aufgabe erfordert nunmehr die Bestimmung des Potentials $\Phi$ einer Linienladung $Q^{\prime}=m Q$ im Ursprung der $x_{2}-y_{2}$-Ebene, für das auf den waagrechten Seiten des in Abb. 3 gezeichneten Rechtecks die Randbedingung $\Phi=0$ und auf den senkrechten Seiten die Randbedingung $\partial \Phi / \partial x_{2}=0$ vorgeschrieben ist. Diese Forderungen können durch Anbringen von unendlich vielen doppeltperiodisch angeordneten Spiegelladungen erfüllt werden (s. Abb. 4). Das komplexe Potential $Z$ dieser Anordnung ist das gleiche wie für eine Flüssigkeitsströmung in einem von undurchlässigen Wänden begrenzten Kanal, in dessen Mitte sich zwei Systeme von unendlich vielen äquidistanten Quellen und Senken befinden [Abstand jeweils $\alpha=2 m \ln (b / a)$ ], die gegeneinander um $\beta=2 m \ln (b / c)$ versetzt sind (Abb. 5). Die komplexen Potentiale dieser Strömungen $^{5}$ lassen sich mit Hilfe von Jacobischen $\vartheta$-Funktionen darstellen ${ }^{6}$. Für die in Abb. 5 dargestellte Strömung lautet das komplexe Potential

$$
\begin{array}{r}
Z=Q^{\prime}\left[\ln \vartheta_{1}\left(\frac{z_{2}}{2 \pi} \mid \tau\right)-\ln \vartheta_{1}\left(\frac{z_{2}+i \beta}{2 \pi} \mid \tau\right)\right] \\
+i Q_{1} z_{2}+Q_{2} .
\end{array}
$$

Dabei ist

$\vartheta_{1}(u \mid \tau)=i \sum_{n=-\infty}^{n=+\infty}(-1)^{n} e^{i \pi \tau\left(n-\frac{1}{2}\right)^{2}} e^{i \pi(2 n-1) u}$

und

$$
\tau=i \frac{m}{\pi} \ln (b / a)=i \frac{a}{2 \pi}
$$

das Periodenverhältnis der doppeltperiodischen Quellenanordnungen. Das Glied $i Q_{1} z_{2}$ stellt (für reelles $Q_{1}$ ) eine Strömung parallel zur $y_{2}$-Achse dar. Bestimmt man $Q_{1}$ und $Q_{2}$ so, daß das Potential an den vorgeschriebenen $y_{2}$-Werten Null wird, so erhält man

$$
Q_{2}=-\frac{1}{2} \beta Q_{1}
$$

$\frac{1}{2} a Q_{1}$

$=Q^{\prime}\left[\ln \vartheta_{1}\left(\frac{i m \ln (c / a)}{2 \pi} \mid \tau\right)-\ln \vartheta_{1}\left(\begin{array}{c}i m \ln \frac{b^{2}}{a c} \\ 2 \pi\end{array}\right)\right]$.

5 Siehe z. B. A. B e tz, Konforme Abbildung, X. Abschnitt, Springer-Verlag, Berlin-Göttingen-Heidelberg 1948.

6 In der Mathematik werden (insbesondere bei der Behandlung der elliptischen Funktionen) vier $\vartheta$-Funktionen $\vartheta_{i}(i=1, \ldots, 4)$ betrachtet. Obwohl sich alle vier Funktionen ineinander transformieren lassen, empfiehlt sich die Verwendung verschiedener Funktionen aus Gründen der praktischen Rechnung.
Die Bestimmung von $Q^{\prime}$ aus der Potentialdifferenz $\Phi_{0}$ zwischen Zähldraht und Zylindern werden wir erst im nächsten Paragraphen vornehmen. Hiervon abgesehen, wird durch die Gln. (4), (5) und (8) das elektrostatische Potential für unser idealisiertes Problem explizit angegeben.

\section{§3. Die praktische Feldbestimmung}

Tabellen für die $\vartheta$-Funktionen eines komplexen Arguments liegen unseres Wissens in der Literatur

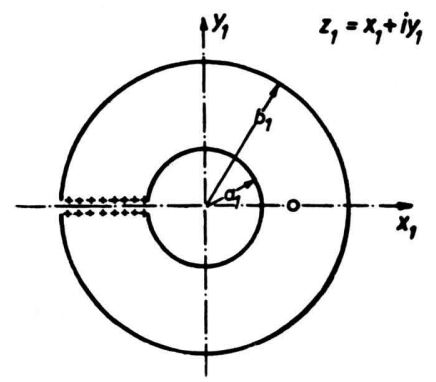

Abb. 2. Konforme Abbildung auf die Ebene $z_{1}=(z / c)^{m}$.

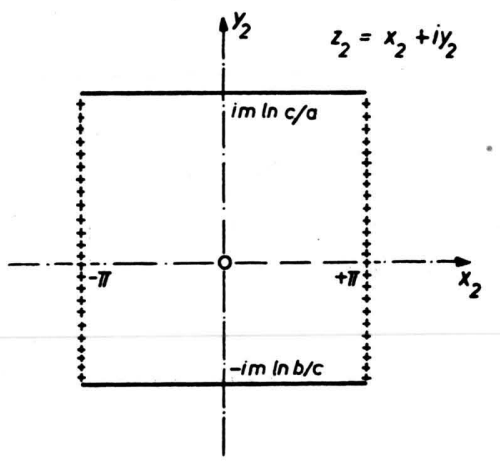

Abb. 3. Konforme Abbildung auf die Ebene $z_{2}=-i \ln z_{1}$ $=-i m \ln (z / c)$.

nicht vor. Ein Grund dafür ist wohl, daß für eine derartige Tabellierung kein praktisches Bedürfnis besteht, da die Konvergenz der unendlichen Reihen für die $\vartheta$-Funktionen hervorragend ist, und da man durch Verwendung der Transformationseigenschaften dieser Funktionen stets erreichen kann, daß die betreffenden Reihen für die praktische Rechnung schon nach wenigen Gliedern abgebrochen werden dürfen ${ }^{7}$. Z. B. läßt es sich stets erreichen, daß der für die gute Konver-

7 Wegen der im folgenden benützten Transformationseigenschaften und Reihenentwicklungen $\operatorname{der} \vartheta$-Funktionen sei auf F. Oberhettinger u. W. Magnus, Anwendungen der elliptischen Funktionen in Physik und Technik, Springer-Verlag, Berlin, Göttingen, Heidelberg 1950, und auf F. Tricomi, Elliptische Funktionen, Akad. Verl.-Ges. Leipzig 1948, verwiesen. 
genz der Reihe Gl. (5) verantwortliche Parameter $-i \tau=m / \pi \ln (b / a)$ größer oder gleich eins ist. Im vorliegenden Beispiel ist

$$
-i \tau=0,339 m=2,018,
$$

so daß man auf den Übergang zu einem Periodenverhältnis $-1 / \tau$ verzichten kann. Dagegen ist es im-

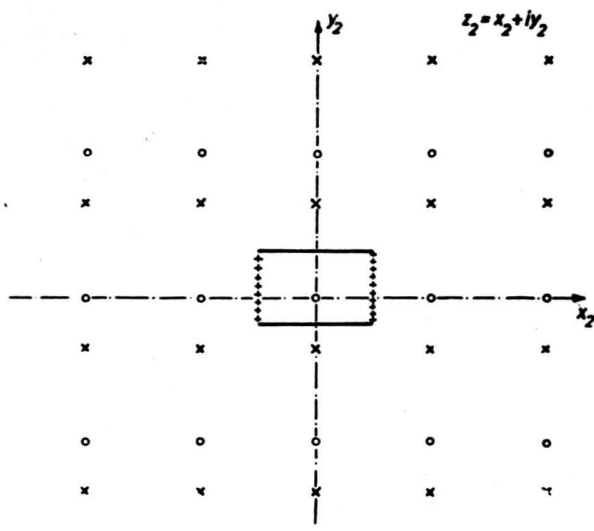

Abb. 4. Erfüllung der Randbedingungen in der $z_{2}$-Ebene durch ein doppelt periodisches System von Quellen $\bigcirc$ und Senken $x$.

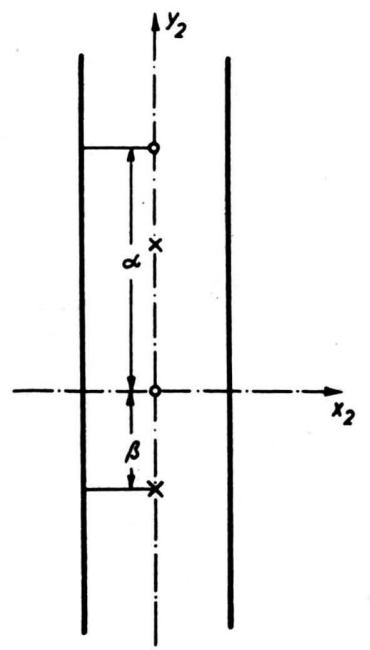

Abb. 5. Äquivalente Kanalströmung. $\alpha=2 m \ln (b / a)=\mathrm{Ab}-$ stand Quelle-Quelle $=$ Abstand Senke-Senke, $\beta=2 m \ln (b / c)=$ Abstand Senke-Quelle.

mer dann, wenn (wie im vorliegenden Fall) $c$ in der Nähe des geometrischen Mittels $c_{0}=(a b)^{1 / 2}$ der Drahtradien $a$ und $b$ liegt, nützlich, im zweiten Glied der Gl. (5) von der $\vartheta_{1}$-Funktion mit Hilfe der Transformationsformel

$$
\vartheta_{1}\left(u+\frac{1}{2} \tau\right)=i \vartheta_{4}(u \mid \tau) e^{-\frac{i \pi}{2}\left(2 u+\frac{1}{2} \tau\right)}
$$

zu der durch

$$
\vartheta_{4}(u \mid \tau)=\sum_{n=-\infty}^{n=+\infty} e^{i \pi \tau n^{2}} e^{i \pi u(2 n+1)}
$$

definierten $\vartheta_{4}$-Funktion überzugehen. Damit lautet das komplexe Potential

$$
\begin{aligned}
Z=Q^{\prime} & \left\{\ln \vartheta_{1}\left(\frac{z_{2}}{2 \pi} \mid \tau\right)-\ln \vartheta_{4}\left(\frac{z_{2}+i \beta}{2 \pi}-\frac{1}{2} \tau \mid \tau\right)-\frac{\beta}{2}\right\} \\
& -\frac{\pi i}{2} Q^{\prime}\left(1-\frac{z_{2}}{\pi}+\frac{\tau}{2}\right)+i Q_{1} z_{2}+Q_{2} .
\end{aligned}
$$

Die Reihen der in Gl. (12) auftretenden Funktionen konvergieren für die vorliegenden Parameterwerte besser als diejenigen in Gl. (5); man kann mit ihrer Hilfe mit verhältnismäßig geringem Rechenaufwand das Potential $\Phi$ in jedem Punkt des Feldes berechnen.

Zur Bestímmung des Zähldrahtpotentials $\Phi_{0}$ beachten wir, daß in der Nähe der Zähldrähte $z_{2} \approx 0$ ist. Wir dürfen daher im Sinne der in $\$ 1$ als zweite genannten Idealisierung in allen Gliedern der Gl. (5) mit Ausnahme des ersten $z_{2}=0$ setzen. Da dieses erste Glied der Gl. (5) an der Stelle $z_{2}=0$ eine logarithmische Singularität besitzt, formen wir es folgendermaßen um:

$$
\begin{aligned}
Q^{\prime} \ln \vartheta_{1}\left(\frac{z_{2}}{2 \pi} \mid \tau\right) \approx Q^{\prime} & \ln \left[\vartheta_{1}{ }^{\prime}(0 \mid \tau) \frac{z_{2}}{2 \pi}\right] \\
& =Q^{\prime}\left[\ln \vartheta_{1}{ }^{\prime}+\ln \frac{z_{2}}{2 \pi}\right] .
\end{aligned}
$$

Dabei ist

$$
\begin{aligned}
& \vartheta_{1}{ }^{\prime}=\left.\frac{\mathrm{d} \vartheta_{1}(u \mid \tau)}{\mathrm{d} u}\right|_{u=0} \\
& =2 \pi q^{1 / 4}\left(1-3 q^{2}+5 q^{6}-\cdots\right)
\end{aligned}
$$

mit

$$
q=e^{i \pi \tau}=(a / b)^{m}
$$

eine leicht berechenbare Funktion von $\tau . \vartheta_{1}{ }^{\prime}$ und $q$ sind bei $\mathrm{H}$ a y a $\mathrm{s} \mathrm{hi}^{8}$ als Funktion der später einzuführenden Variablen $k^{2}$ tabelliert.

Für den Übergang zur $x$ - $y$-Ebene setzen wir

$$
z=c(1+w / c)
$$

dann ist für $w / c \ll 1$,

$$
z_{2}=-i m w / c .
$$

In der unmittelbaren Umgebung des Drahtes ist (bei Vernachlässigung höherer Potenzen von $m w / c$ )

8 K. H a y a shi, Fünfstellige Funktionentafeln, Springer, Berlin 1931, S. 147-154. 


\begin{tabular}{|c|c|c|c|c|c|c|c|c|}
\hline$q$ & $-i \tau=\frac{a}{2 \pi}$ & $a$ & $\beta / 2 \pi$ & $\beta$ & $k^{2}$ & $\ln \frac{m d}{2 c}$ & $\ln \frac{\vartheta_{1}}{2 \pi}$ & $\ln \left|\vartheta_{1}\left(\frac{i \beta}{2 \pi} \mid \tau\right)\right|$ \\
\hline $1,683 \cdot 10^{-3}$ & 2,018 & 12,776 & 0,772 & 4,763 & 0,0265 & $-4,5 \varepsilon 0$ & $-1,584$ & $-0,776$ \\
\hline
\end{tabular}

Tab. 1.

das Potential gegeben durch

$$
\begin{aligned}
\Phi=\mathfrak{R e}\left\{Q^{\prime} \ln \left(\frac{-i m w}{c}\right)+Q^{\prime} \ln \frac{\vartheta_{1}{ }^{\prime}}{2 \pi}\right. \\
\quad-Q^{\prime} \ln \vartheta_{1}\left(\frac{i \beta}{2 \pi} \mid \tau\right)+Q_{2 .} .
\end{aligned}
$$

In dieser Näherung sind die Äquipotentialfächen in der Nähe der Zähldrahtmittelpunkte die Kreise $|w|=$ const. Für $|w|=1 / 2 d$ erhält man das Zähldrahtpotential

$\Phi_{0}=Q^{\prime}\left\{\ln \frac{m d}{2 c}+\ln \frac{\vartheta_{1}}{2 \pi}-\ln \left|\vartheta_{1}\left(\frac{i \beta}{2 \pi} \mid \tau\right)\right|+Q_{2}\right.$.

In Tab. 1 stellen wir die bei der numerischen Auswertung der vorstehenden Formeln auftretenden Zahlenwerte zusammen.

Um die späteren Rechnungen mit elliptischen Funktionen zu erleichtern, wurde das Quadrat des Moduls $k$ dieser Funktionen, das man als elliptische Modulfunktion

$$
k^{2}=k^{2}(\tau)
$$

bezeichnet ${ }^{9}$, eingeführt.

Setzt man die Zahlenwerte der Tab. 1 in die Gl. (8) und (19) ein und löst nach den Konstanten $Q^{\prime}, Q_{1}$ und $Q_{2}$, die sämtlich die Dimension des elektrischen Potentials haben, auf, so ergibt sich

$$
\begin{aligned}
& Q^{\prime}=-0,198 \quad \Phi_{0}, \\
& Q_{1}=+0,0757 \Phi_{0}, \\
& Q_{2}=-0,180 \Phi_{0} .
\end{aligned}
$$

Von besonderem praktischen Interesse ist die Kenntnis des elektrostatischen Feldes in der Nähe der Geraden $z_{2}= \pm \pi$. d. h. in den Mittelebenen zwischen je zwei Zähldrähten, da dort eine singuläre Linie mit der Feldstärke $\mathfrak{\xi}=0$ auftritt. Für die Verwendbarkeit des Zählrohres ist es von großer Bedeutung, ob in der Umgebung dieser feldfreien Linie der Feldstärkengradient genügend groß ist.

Um diese Verhältnisse bequem studieren zu können, führen wir eine neue Variable $z_{3}$ durch die Gleichungen

$$
\begin{aligned}
& z_{3}=-i\left(z_{2}-\pi\right), \\
& z_{2}=i z_{3}+\pi
\end{aligned}
$$

ein, so daß einer der obengenannten Geraden die reelle $z_{3}$-Achse entspricht. Wegen der Transformationsformel

$$
\begin{aligned}
& \vartheta_{1}\left(u+\frac{1}{2} \mid \tau\right)=\vartheta_{2}(u \mid \tau) \\
& =\sum_{n=-\infty}^{\infty} \exp \left(i \pi \tau\left(n+\frac{1}{2}\right)^{2}\right) \exp (i \pi(2 n+1) u)
\end{aligned}
$$

lautet das komplexe Potential jetzt

$$
\begin{aligned}
Z=Q^{\prime}\left\{\ln \vartheta_{2}\left(\frac{i z_{3}}{2 \pi} \mid \tau\right)-\right. & \left.\ln \vartheta_{2}\left(\frac{i\left(z_{3}+\beta\right)}{2 \pi} \mid \tau\right)\right\} \\
& -Q_{1} z_{3}+i Q_{1} \pi+Q_{2} .
\end{aligned}
$$

Statt der elektrischen Feldstärke $\mathfrak{F}$ berechnen wir den an der $y$-Achse gespiegelten Vektor

$$
-\mathfrak{F}^{*}=\frac{\mathrm{d} Z}{\mathrm{~d} z}=\frac{\mathrm{dZ}}{\mathrm{d} z_{3}} \frac{\mathrm{d} z_{3}}{\mathrm{~d} z}=-\frac{\mathrm{dZ}}{\mathrm{d} z_{3}} \frac{m}{z} .
$$

Hierbei ist

$$
\frac{\mathrm{dZ}}{\mathrm{d} z_{3}}=\frac{i Q^{\prime}}{2 \pi}\left\{\frac{\vartheta_{2}^{\prime}\left(\frac{i z_{3}}{2 \pi} \mid \tau\right)}{\vartheta_{2}\left(\frac{i z_{3}}{2 \pi} \mid \tau\right)}-\frac{\vartheta_{2}^{\prime}\left(\frac{i\left(z_{3}+\beta\right)}{2 \pi}\right)}{\vartheta_{2}\left(\frac{i\left(z_{3}+\beta\right)}{2 \pi}\right)}\right\}-Q_{1}
$$

Die räumliche Änderung der elektrischen Feldstärke bekommt man aus der Gleichung

$$
-\frac{\mathrm{d} 巨^{*}}{\mathrm{~d} z}=\frac{\mathrm{d}^{2} Z}{\mathrm{~d} z^{2}}=\frac{m}{z^{2}}\left(\frac{\mathrm{d}^{2} Z}{\mathrm{~d} z_{3}{ }^{2}} m+\frac{\mathrm{d} Z}{\mathrm{~d} z_{3}}\right)
$$

mit

$$
\frac{\mathrm{d}^{2} Z}{\mathrm{~d} z_{3}{ }^{2}}=-\frac{Q^{\prime}}{(2 \pi)^{2}} \frac{\mathrm{d}^{2}}{\mathrm{~d} u^{2}} \ln \left\{\frac{\vartheta_{2}(u \mid \tau)}{\vartheta_{2}\left(u+\frac{i \beta}{2 \pi} \mid \tau\right)}\right\} ; u=\frac{i z_{3}}{2 \pi} .
$$

Da wir uns im Augenblick besonders für reelle $z_{3}$ interessieren, ist es zweckmäßig, in Gl. (26) dạs unvollständige elliptische Integral 2. Gattung

$$
E(\varphi, k)=E(v \mid k)=\int_{0}^{q}\left(1-k^{2} \sin ^{2} \varphi\right)^{1 / 2} \mathrm{~d} \varphi,
$$

aufgefaßt als Funktion des unvollständigen elliptischen Integrals 1. Gattung

$$
v=F(\tau, k)=\int_{0}^{q}\left(1-k^{2} \sin ^{2} \tau\right)^{-1 / 2} \mathrm{~d} \tau,
$$

9 J a hnke-Emcle, Tafeln höherer Funktionen, 4. Aufl., Teubner, Leipzig 1948, S. 44, 48, 49. 
einzuführen. $E(v \mid k)$ hängt durch die Gleichung

$$
E(v \mid k)=\frac{1}{2 \mathbf{K}} \frac{\vartheta_{4}{ }^{\prime}\left(\frac{v}{2 \mathbf{K}} \mid \tau\right)}{\vartheta_{4}\left(\frac{v}{2 \mathbf{K}} \mid \tau\right)}+\frac{\mathbf{E}}{\mathbf{K}} v
$$

mit der $\vartheta_{4}$-Funktion zusammen, welche wiederum durch die Transformation

$$
\vartheta_{2}(u \mid \tau) \sqrt{\frac{\tau}{i}} e^{i \frac{\pi}{\tau} u^{2}}=\vartheta_{4}\left(\frac{u}{\tau} \mid-\frac{1}{\tau}\right)
$$

mit $\vartheta_{2}(u \mid \tau)$ verknüpft ist.

Dem Übergang von einer $\vartheta$-Funktion mit dem Periodenverhältnis $\tau \mathrm{zu}$ einer solchen mit dem Periodenverhältnis $-1 / \tau$ entspricht dem Übergang vom Modul $k$ zum komplementären Modul

$$
k^{\prime}=\left(1-k^{2}\right)^{1 / 2} .
$$

Entsprechend geht das vollständige elliptische Integral 1. Gattung

über in

$$
\mathbf{K}=F(\pi / 2, k)
$$

$$
\mathbf{K}^{\prime}=\mathbf{K}\left(k^{\prime}\right)=F\left(\pi / 2, k^{\prime}\right),
$$

und das vollständige elliptische Integral 2. Gattung

über in

$$
\mathbf{E}=E(\pi / 2, k)=E(\mathbf{K} \mid k)
$$

$$
\mathbf{E}^{\prime}=\mathbf{E}\left(k^{\prime}\right)=E\left(\pi / 2, k^{\prime}\right)=E\left(\mathbf{K}^{\prime} \mid k^{\prime}\right) .
$$

Mit diesen Funktionen wird

$$
\begin{array}{r}
\frac{\mathrm{d} Z}{\mathrm{~d} z_{3}{ }^{2}}=\frac{Q^{\prime} \mathbf{K}}{\pi}\left\{E\left(\frac{z_{3} \mathbf{K}}{\pi} \mid k^{\prime}\right)-E\left(\frac{\left(z_{3}+\beta\right) \mathbf{K}}{\pi} \mid k^{\prime}\right)\right\} \\
+\frac{\beta \mathbf{K} Q^{\prime}}{2 \mathbf{K}^{\prime} \pi}\left\{\frac{2 \mathbf{K} \mathbf{E}^{\prime}}{\pi}-1\right\}-Q_{1}
\end{array}
$$

und

$\frac{\mathrm{d}^{2} Z}{\mathrm{~d} z_{3}{ }^{2}}=Q^{\prime}\left(\frac{\mathbf{K}}{\pi}\right)^{2} k^{\prime 2}\left\{\operatorname{sn}^{2}\left(\frac{\left(z_{3}+\beta\right) \mathbf{K}}{\pi}, k^{\prime}\right)-\operatorname{sn}^{2}\left(\frac{z_{3} \mathbf{K}}{\pi}, k^{\prime}\right)\right\}$

$\operatorname{sn}(v, k)=\sin \varphi$ ist eine der Jacobischen elliptischen Funktionen [ $v$ und $\varphi$ hängen nach Gl. (30) zusammen].

Wir haben mit Hilfe der Legendreschen Tafeln ${ }^{10}$ die elektrische Feldstärke in den Symmetrieebenen zwischen den Zähldrähten nach Gl. (36) für die in der Tab. 1 wiedergegebenen Zahlenwerte numerisch berechnet (Abb. 6). Im feldfreien Punkt ist $|z|=1,08 c$; er liegt also ein wenig außerhalb des Zähldrahtkreises $|z|=c$. Ist $\Psi$ der Polarwinkel, also $z=|z| e^{i \psi}$ der Ortsvektor dieses Punktes, so ist nach Gl. (27) und (37)

$$
\frac{\mathrm{d}^{*}}{\mathrm{~d} z}=1,42 \cdot e^{-i 2 \psi} \frac{\Phi_{0}}{c^{2}} .
$$

10 A. M. L e g e n d r e, Tafeln der elliptischen Normalintegrale, Konrad Wittwer, Stuttgart 1931.
Man kann aus Abb. 6 einige praktische Folgerungen ziehen: Es hätte keinen Sinn, zwischen Innenund Außenzylinder ein zusätzliches zylindersymmetrisches elektrisches Feld anzulegen, weil sich dadurch die Steilheit der $\mathfrak{F}$-Kurve im feldfreien Punkt nicht wesentlich erhöhen läßt. Dagegen ist es vielleicht zweckmäßig, das Verhältnis $c / a$ etwas kleiner als im durchgerechneten Beispiel zu machen, da dann das elektrische Feld wohl noch steiler als in Abb. 6 gezeichnet von positiven zu negativen Werten übergeht. Grenzen hierfür sind natürlich dadurch gesetzt, daß die maximale Feldstärke einen gewissen Betrag nicht

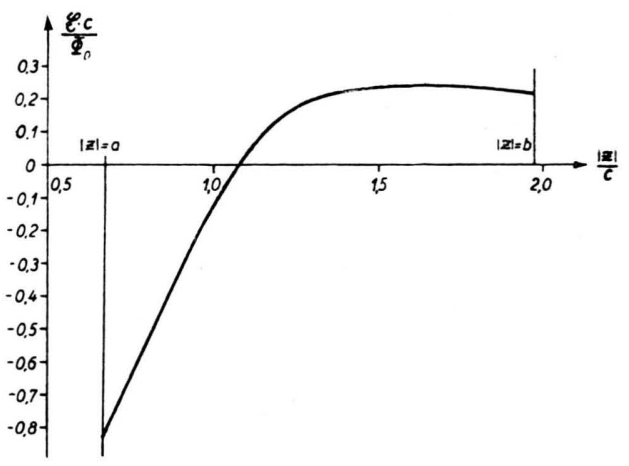

Abb. 6. Verlauf der elektrischen Feldstärke $\mathfrak{F}$ in den Symmetrieebenen zwischen den Zähldrähten als Funktion des Radius $|z|$.

übersteigen darf. Unser Zahlenbeispiel ist für diese Überlegungen jedoch nicht ganz stichhaltig, da wir uns ja auf feste Verhältnisse $c / a$ und $b / c$ beschränkt haben.

$\S$ 4. Berücksichtigung des endlichen Zähldrahtdurchmessers

Die Vernachlässigung von $\delta=m d / 2 c$ neben Zahlen von der Größenordnung 1 , die wir in $\$ 3$ zur Bestimmung des Zähldrahtpotentials vorgenommen hatten, ist im vorliegenden Beispiel erlaubt, da hier $\delta=0,01$ ist. Wir geben deshalb nur das Prinzip an, nach dem man, falls erforderlich, höhere Potenzen von $\delta$ berücksichtigen kann. Wir suchen zu diesem Zwecke eine Näherungslösung des strengen Feldbestimmungsproblems, das nicht im Zähldrahtmittelpunkt eine vorgeschriebene Singularität wie beim idealisierten Problem, sondern auf dem Kreiszylinder $|w|=1 / 2 d$ konstanten Realteil $\Phi=\Re e \mathbf{Z}=\Phi_{0}$ der komplexen Potentialfunktion $\mathbf{Z}$ fordert. Das Abbild von $|w|=1 / 2 d$ ist in der $z_{2}$-Ebene eine komplizierte geschlossene Kurve um den Nullpunkt. Für die Lösung des strengen Potentialproblems dürfen wir alle 
analytischen Funktionen heranziehen, die im Zwischengebiet zwischen dieser Kurve und dem in Abb. 3 gezeichneten Rechteck regulär sind und welche die auf den Rechteckseiten vorgeschriebene Randbedingung (s. § 2) erfüllen. Dies sind jedoch gerade die Ableitungen beliebiger Ordnung $\frac{\mathrm{d}^{n} Z}{\mathrm{~d} z_{2}{ }^{n}}$ der Funktion $Z=$ $Z\left(z_{2}\right)$ Gl. (5). Man kann also das komplexe Potential $\mathbf{Z}$ in eine Multipolreihe

$$
\mathbf{Z}=\sum_{n=0}^{\infty} c_{n} \frac{\mathrm{d}^{n} \mathrm{Z}}{\mathrm{d} z_{2}{ }^{2}}
$$

mit komplexen Koeffizienten $c_{n}$ entwickeln. Die $c_{n}$ sind so zu bestimmen, daß

$$
\mathfrak{R e} \mathbf{Z}=\Phi_{0} \quad \text { für } \quad|w|=\frac{1}{2} d
$$

ist. Bei der praktischen Durchführung wird man natürlich statt mit der unendlichen Reihe Gl. (39) mit einem Aggregat von endlich vielen Gliedern rechnen und zur Bestimmung der Konstanten aus Gl. (40) die entsprechenden höheren Potenzen von $\delta$ vernachlässigen.

Für Anregungen und Ratschläge habe ich Herrn Prof. Dr. E. F u e s, Herrn Prof. Dr. E. S c h o p p e r und Herrn cand. phys. H. Jür ge n s e n zu danken. Ich freue mich, diese Arbeit Herrn Prof. Dr. E. R e ge n e r zu seinem siebzigsten Geburtstage als einen Beitrag zum wissenschaftlichen Arbeitsprogramm seines Instituts widmen zu können.

\title{
Untersuchungen an Geiger-Müller-Zählern mit mehreren Zähldrähten
}

\author{
Von H. Jürgensen und H. M. Weiss \\ Aus dem Physikalischen Institut der Technischen Hochschule Stuttgart \\ und der Forschungsstelle für Physik der Stratosphäre in der Max-Planck-Gesellschaft Weißenau \\ (Z. Naturforschg. 6 a, 694-698 [1951]; eingegangen am 9. August 1951)
}

\section{Herrn Professor Erich Regener zum 70. Geburtstag}

Die Zähleigenschaften von Auslösezählern mit mehreren Zähldrähten zwischen ebenen bzw. konzentrisch gekrümmten Platten werden im Hinblick auf die Verwendung als Großflächenzähler untersucht. Die Entladungen sind nicht auf den Zählbereich der einzelnen Zähldrähte lokalisiert; das Übergreifen der Entladung wird untersucht. Als praktische Anwendung wird eine Antikoinzidenz-Abschirmung durch einen Mehrdraht-Kreisringzähler gegenüber einem Zentralzählrohr zur Zählung geringer Intensitäten beschrieben.

Z ur Anwendung auf spezielle Probleme wurde früher von E. R e g e n e r die Untersuchung von Zählrohren nichtzylindrischer Form angeregt ${ }^{1}$. Im folgenden berichten wir über Messungen an Auslösezählern mit mehreren Zähldrähten zwischen parallelen ${ }^{2}$ bzw. konzentrisch gekrümmten Platten ${ }^{3}$.

\section{Zähleigenschaften eines Mehrdraht- Parallelplatten-Zählers}

Der Aufbau des Zählers ist aus Abb. 1 zu ersehen: In der Mittelebene zweier paralleler Metallplatten sind mehrere äquidistante Zähldrähte gespannt. Die Anordnung entspricht einer in einem einzigen Zähler zusammengefaßten Zählrohrlage. Ein solcher Mehrdrahtzähler bietet gewisse Vorteile als Großflächenzähler oder als Antikoinzidenzabschirmung.

Der für die Zähleigenschaften maßgebende Feld-

1 E. S a u r, Diplomarbeit, Stuttgart 1934.

2 H. M. W e i $\beta$, Diplomarbeit, Stuttgart 1950. verlauf unserer Anordnung ist für parallele Platten noch einfach berechenbar. Für konzentrisch gekrümmte Platten (sog. Kreisringzähler) hat S e e g e $\mathrm{r}^{4}$ eine geschlossene Lösung angegeben. Der experimentell im elektrolytischen Trog ermittelte Feldverlauf ergab das in Abb. 1 gezeichnete Bild. Von den Kathodenplatten ausgehend, verläuft das Feld homogen bis zu einer Entfernung von der Drahtreihe, die etwa dem halben Drahtabstand entspricht. Die Entfernung der Zähldrähte darf also höchstens so groß sein, daß von einem senkrecht durch das Zwischengebiet fliegenden Teilchen im homogenen Feldbereich noch mindestens ein Ionenpaar erzeugt wird. In der untersuchten Anordnung war $d=9 \mathrm{~mm}, D=20 \mathrm{~mm}$ (Abb. 1). Bei der Abtastung des Zählers an verschiedenen Stellen mit einem schmalen $\gamma$-Strahlbündel erhält man über den gesamten Zähler hinweg bis auf die unmittelbaren Randgebiete eine konstante Impulszahl.

3 H. J ür g e n s e n, Diplomarbeit, Stuttgart 1951.

4 A. S e e g e r, Z. Naturforschg. 6a, 688 [1951]. 